Solid State Communications, Vol. 29, pp. 523-526.

Pergamon Press Ltd. 1979. Printed in Great Britain.

\title{
PIEZOBIREFRINGENCE IN GaP AND InP
}

F. Canal, ${ }^{*}$ M. Grimsditch and M. Cardona

Max-Planck-Institut für Festkörperforschung, 7 Stuttgart 80, West Germany

(Received 16 November 1978 by M. Cardona)

\begin{abstract}
We report measurements of piezobirefringence in $\mathrm{GaP}$ and InP in the region below the direct gap. For $\mathrm{GaP}$ this includes the region above the indirect gap where the sample is opaque. The experimental data are compared with theoretical calculations of the contributions to the piezobirefringence arising from the $E_{0}, E_{1}$ and $E_{2}$ transitions.
\end{abstract}

\section{INTRODUCTION}

THE INVESTIGATION of stress induced birefringence in solids is an old topic of crystal optics [1]. Only relatively recently, however, has a systematic investigation of the dispersion of the piezooptical constants of a number of materials been undertaken [2] . Prominent among these materials are the zinc-blende and diamond-like semiconductors. Due to the rather detailed understanding of the microscopic mechanisms underlying their optical properties it is possible to obtain for these materials theoretical expressions for the piezooptical constants and their dispersion. Data in the region of transparency have been presented for $\mathrm{Ge}$, $\mathrm{Si}$ [3], AlSb [4], GaAs [3], GaSb, InAs, InSb [5], $\mathrm{ZnS}, \mathrm{ZnSe}, \mathrm{ZnTe}, \mathrm{CdTe}$ [6], $\mathrm{CuCl}, \mathrm{CuBr}$, and $\mathrm{CuI}$ [7]. More recently a technique has been developed to investigate the piezo-optical constants above the absorption edge and applied to silicon [8]. In this paper we complete existing data by reporting the dispersion of the birefringence induced by a [111] and a [100] stress for InP, a direct gap material, in the region of transparency and for $\mathrm{GaP}$, an indirect gap material, all the way up to the lowest direct gap.

From these and previous measurements the following picture emerges: the piezooptical constants in indirect gap materials ( $\mathrm{Si}, \mathrm{GaP}, \mathrm{AlSb}$ ) are not strongly dispersive as this gap is approached. They show, however, a strong dispersion in all materials of the family as the lowest direct gap is approached. The overall dispersion observed can be synthesized as the sum of three dispersion mechanisms. The $\Gamma_{15} \rightarrow \Gamma_{1}$ gap $\left(E_{0}\right)$, the $\Lambda_{3} \rightarrow \Lambda_{1}$ gap $\left(E_{1}\right)$ and a gap at higher energies $\left(E_{2}\right)$ which corresponds to the maximum of $\epsilon_{2}$ in the material. The $E_{0}$ contributions have the same sign for [100] and for [111] stress and opposite sign to the sum of the $E_{1}$ and $E_{2}$ contributions. The $E_{1}$ and $E_{0}$

\footnotetext{
* Present address: Escuela Técnica Superior de Ingenieros de Telecommunicación, Barcelona, Spain.
}

contributions have the same sign for [100] stress and opposite sign for [111] stress. The $E_{0}$ and $E_{1}$ contributions can be expressed analytically in terms of deformation potentials which are in semi-quantitative agreement with those found with other methods.

\section{EXPERIMENTAL}

The experimental method used to determine the piezooptical constants in the region where the sample is transparent has been described in the literature [9]. We also used a new technique that extends measurements into the region where the sample is opaque. This technique has been sufficiently described [8] and the details will not be repeated here. The measurements were made at room temperature using the stress apparatus described in [10].

In the transparent region of the samples the combinations of piezo-optical constants $\left(\pi_{11}-\pi_{12}\right)$ and $\pi_{44}$ were determined by stressing single crystal parallelepipeds along the [100] and [111] directions, respectively.

In the region where the samples are opaque we used the scattering geometries labelled (a) and (b) in [8] to determined the combinations $\pi_{44}$ and $\left(\pi_{11}-\pi_{12}\right)$, respectively. The experimentally determined depolarization of the Raman line as given by the ratio of intensities of the non-allowed to allowed polarizations $(\beta)$ was fitted to an expression of the form:

$$
\beta=\frac{1}{1+\frac{1}{2(A X)^{2}}}+C,
$$

where $X$ is the applied stress and

$A=\frac{\pi n^{3} \pi_{i j}}{\lambda\left(\alpha_{i}+\alpha_{i}\right)}$.

In equation (2), $n$ and $\lambda$ are the average refractive index and the wavelength for the incident and scattered light, 


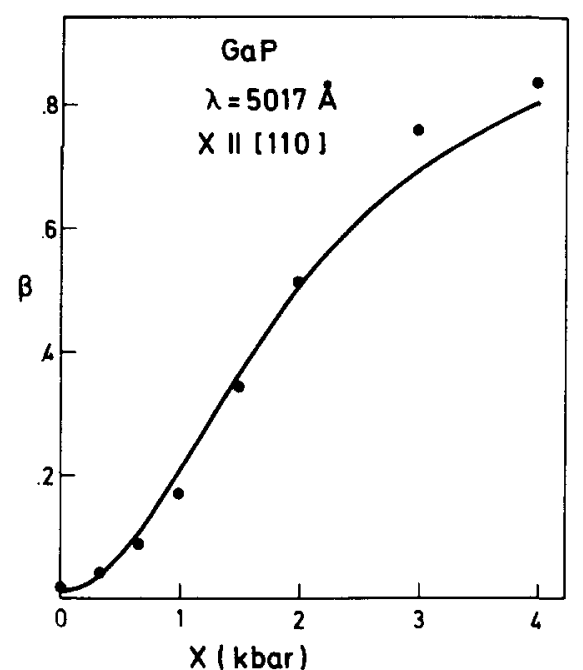

Fig. 1. Depolarization ratio $(\beta)$ of the LO phonon of $\mathrm{GaP}$ observed from a (001) face as a function of applied stress along [110]. The wavelength of the incident radiation is $5017 \AA$.

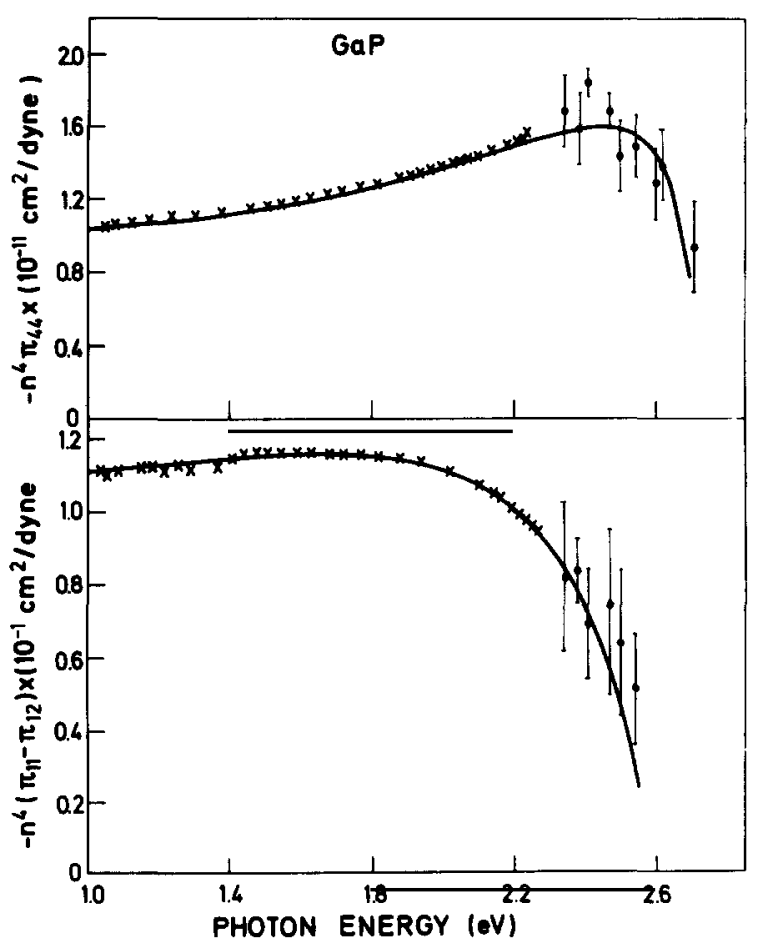

Fig. 2. Measured values of $n^{4} \pi_{44}$ and $n^{4}\left(\pi_{11}-\pi_{12}\right)$ for $\mathrm{GaP}$ as a function of photon energy. The crosses indicate values determined by conventional techniques where the sample is transparent, the dots are values obtained from the depolarization of the Raman spectra in the region where the sample is opaque. The full line represents a fit to the experimental values due to the various contributions to the birefringence of the various critical points in the band structure.

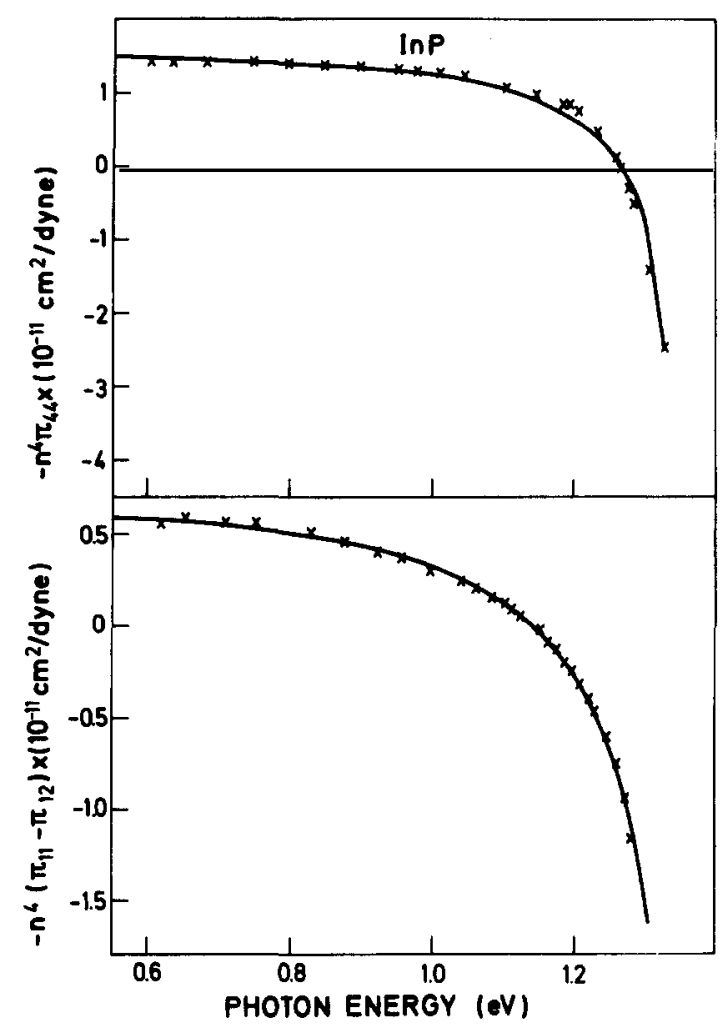

Fig. 3. Measured values of $n^{4} \pi_{44}$ and $n^{4}\left(\pi_{11}-\pi_{12}\right)$ for InP as a function of photon energy. The meaning of the crosses and full line is the same as in Fig. 2.

$\pi_{i j}$ is $\pi_{44}$ for case (a) and $\pi_{11}-\pi_{12}$ for case (b), $\alpha_{i}$ and $\alpha_{g}$ are the absorption coefficients for the incident and scattered light. The constant $C$ in equation (1) is included in the fit to account for ary leakage (i.e. spurious depolarization) that may occur at zero stress.

\section{RESULTS}

The results obtained in the opaque region for $\beta$ at a wavelength of $5017 \AA$, for stress along [110], by observing the Raman active $L O$ phonon from a (001) face of $\mathrm{GaP}$ are shown in Fig. 1. By fitting equation (1) to these results we obtain $A=0.35 \mathrm{kbar}^{-1}$, which in turn leads to $\pi_{44}=11 \times 10^{-14} \mathrm{~cm}^{2} /$ dyne. In the analysis of our data for $\mathrm{GaP}$ we have used values of the absorption coefficient measured for our crystals. These absorption coefficients are in agreement with the values given in [11]. We have interpolated the refractive index between the values given in [12] and [13].

The values we obtain for $\pi_{i j}$ for $\mathrm{GaP}$ can be compared with those obtained by Dixon [14] at $0.63 \mu \mathrm{m}$ which are $\pi_{44}=-9.8$ and $\pi_{11}-\pi_{12}=-8.4$ in units of $10^{-14} \mathrm{~cm}^{2} /$ dyne. Our values for the same constants are $\pi_{44}=-11.6$ and $\pi_{11}-\pi_{12}=-9.7$ in the same units.

In Figs. 2 and 3 we present our values for $n^{4} \pi_{44}$ and 
$n^{4}\left(\pi_{11}-\pi_{12}\right)$ for GaP and InP, respectively. The crosses were obtained by conventional techniques, the dots from the depolarization of the Raman spectra. For InP we were unable to use the new technique of determining the piezo-optical constants where the sample is opaque. This is due to a combination of reasons; the Raman scattering is extremely weak, which leads to noisy signals. Furthermore, the small penetration depth arising from the direct nature of the gap, leads to small values of $\boldsymbol{A}$ [see equation (2)]. The small depolarization expected, together with the noisy signals made measurements impracticable. In the analysis of the data of InP we have used the refractive indices of [15].

\section{THEORETICAL INTERPRETATION}

The band structures of most of the tetrahedrally bonded semiconductors are well known. It is also known that the main contributions to the dielectric constant arise from the spin-orbit split band at zone center $E_{0}$ and $E_{0}+\Delta_{0}$ the spin-orbit split bands along the $\langle 111\rangle$ directions $E_{1}$ and $E_{1}+\Delta_{1}$, and last but not least a large contribution from higher bands which is customarily labelled [2] $E_{2}$. In the analysis of piezobirefringence in Si, Chandrasekhar et al. [8] have considered only contributions to the piezobirefringence due to the $E_{1}, E_{1}+\Delta_{1}$ and $E_{2}$ gaps. The $E_{0}$ and $E_{0}+\Delta_{0}$ gaps in Si have been neglected because they are almost degenerate with the $E_{1}$ gap and have a much smaller oscillator strength. However, in $\mathrm{GaP}$ and InP, where the $E_{0}$ band lies below $E_{1}$, this contribution can no longer be neglected. We give here the contributions to piezobirefringence arising from the $E_{0}, E_{0}+\Delta_{0}, E_{1}, E_{1}+\Delta_{1}$ and $E_{2}$ gaps $[2,16]$. For each gap we consider the two cases $X \|[100]$ and $X \|[111]$.

$E_{0}$ and $E_{0}+\Delta_{0}$ gap

$$
\begin{gathered}
\left(\frac{\epsilon_{\|}-\epsilon_{\perp}}{X}\right)^{100}=C_{0}\left(-g\left(x_{0}\right)+\frac{4 E_{0}}{\Delta_{0}}\right. \\
\left.\times\left[f(x)-\left(\frac{E_{0}}{E_{0}+\Delta_{0}}\right)^{3 / 2} f\left(x_{0 s}\right)\right]\right\},
\end{gathered}
$$

where $\epsilon_{\|}$and $\epsilon_{1}$ are the dielectric constants for radiation polarized parallel and perpendicular to the applied stress, $x_{0}=\hbar \omega / E_{0}, x_{0 s}=\hbar \omega /\left(E_{0}+\Delta_{0}\right)$ and $g(x)$ and $f(x)$ are defined by

$$
\begin{aligned}
& g(x)=x^{-2}\left[2-(1+x)^{-1 / 2}-(1-x)^{-1 / 2}\right], \\
& f(x)=x^{-2}\left[2-(1+x)^{1 / 2}-(1-x)^{1 / 2}\right] .
\end{aligned}
$$

The constant $C_{0}$ is given by

$C_{0}=\frac{3}{4} \frac{C_{0}^{n}\left(S_{11}-S_{12}\right) b}{E_{0}}$, where $S_{U}$ are the elastic compliance constants and $b$ the deformation potential of the $\Gamma_{15}$ valence bands for [100] stress. The constant $C_{0}^{n}$ is related to the contribution to the real part of the dielectric constant of the $E_{0}$ and $E_{0}+\Delta_{0}$ gaps by

$\epsilon_{r}-1 \simeq C_{0}^{\prime \prime}\left[f\left(x_{0}\right)+0.435 f\left(x_{0 \varepsilon}\right)\right]$.

The equivalent expression for [111] stress is obtained from equation (3) by replacing $C_{0}$ by $C_{0}^{\prime}$ where

$C_{0}^{\prime}=\frac{\sqrt{3} C_{0}^{n} S_{44} d}{8 E_{0}}$

and $d$ is the deformation potential of the $\Gamma_{13}$ valence bands for a [111] stress.

$E_{1}$ and $E_{1}+\Delta_{1}$ gap

$$
\begin{gathered}
\left(\frac{\epsilon_{\|}-\epsilon_{\perp}}{X}\right)^{[100]}=C_{1}\left[x_{1}^{-2} \ln \left(1-x_{1}^{2}\right)\right. \\
\left.-\left(\frac{E_{1}}{E_{1}+\Delta_{1}}\right) x_{18}^{-2} \ln \left(1-x_{1 s}^{2}\right)\right],
\end{gathered}
$$

where $x_{1}=\hbar \omega / E_{1}$ and $x_{1}=\hbar \omega /\left(E_{1}+\Delta_{1}\right)$. The constant $C_{1}$ is defined by

$C_{1}=\frac{16 \sqrt{2}}{3} \frac{D_{3}^{3}\left(S_{11}-S_{12}\right)}{a_{0} \Delta_{1} E_{1}}$,

where $D_{3}^{3}$ is a deformation potential constant and $a_{0}$ the lattice parameter. Also

$$
\begin{gathered}
\left(\frac{\epsilon_{\|}-\epsilon_{\perp}}{X}\right)^{[111]}=C_{1}^{\prime}\left[\left(1-x_{1}^{2}\right)^{-1}\right. \\
\left.+\left(\frac{\underline{E}_{1}}{E_{1}+\Delta_{1}}\right)^{2}\left(1-x_{1 s}^{2}\right)^{-1}\right]
\end{gathered}
$$

and

$C_{1}^{\prime}=\frac{8}{9} \frac{S_{44}\left(D_{1}^{5}+2 \sqrt{2} D_{3}^{5}\right)}{a_{0} E_{1}^{2}}$

$E_{2}$ gap

$\left(\frac{\epsilon_{\|}-\epsilon_{1}}{X}\right)^{[100]}=C_{2}\left(1+2 x_{2}^{2}\right)$,

where $x_{2}=\hbar \omega / E_{2}$ and $C_{2}$ is an experimentally determined parameter. For stresses along [111] equation (13) remains valid with a different value of $C_{2}$, i.e. $C_{2}^{\prime}$.

\section{DISCUSSION}

It is easy to show that the terms $\left[\left(\epsilon_{\|}-\epsilon_{\perp}\right) / X\right]^{[100]}$ and $\left[\left(\epsilon_{\|}-\epsilon_{\perp}\right) / X\right]^{[111]}$ are equal to $-n^{4}\left(\pi_{11}-\pi_{12}\right)$ and $-n^{4} \pi_{44}$, respectively. In Figs. 2 and 3 we give our experimental results for these constants as a function of 
Table 1. Parameters entering the evaluation of the various contributions to birefringence

\begin{tabular}{lllllllllllll}
\hline $\begin{array}{l}E_{0} \\
(\mathrm{eV})\end{array}$ & $\begin{array}{l}\Delta_{0} \\
(\mathrm{eV})\end{array}$ & $\begin{array}{l}E_{1} \\
(\mathrm{eV})\end{array}$ & $\begin{array}{l}\Delta_{1} \\
(\mathrm{eV})\end{array}$ & $\begin{array}{l}E_{2} \\
(\mathrm{eV})\end{array}$ & $\begin{array}{l}a_{0} \\
(\AA)\end{array}$ & $\begin{array}{l}S_{11}-S_{12} \\
\left(\mathrm{~cm}^{2} / \mathrm{dyne}\right)\end{array}$ & $\begin{array}{l}S_{44} \\
\left(\mathrm{~cm}^{2} / \mathrm{dyne}\right)\end{array}$ & $\begin{array}{l}b \\
(\mathrm{eV})\end{array}$ & $\begin{array}{l}d \\
(\mathrm{eV})\end{array}$ & $\begin{array}{l}D_{3}^{3} \\
(\mathrm{eV})\end{array}$ & $\begin{array}{l}D_{1}^{\mathrm{s}}+2 \sqrt{2} D_{3}^{\mathrm{s}} \\
(\mathrm{eV})\end{array}$ \\
\hline $\mathrm{GaP}$ & $2.78^{\mathrm{a}}$ & $0.12^{\mathrm{a}}$ & $3.69^{\mathrm{a}}$ & $0.08^{\mathrm{a}}$ & $5.3^{\mathrm{a}}$ & $5.45^{\mathrm{b}}$ & $1.27 \times 10^{-12 \mathrm{c}}$ & $1.42 \times 10^{-12 \mathrm{c}}$ & $-1.8^{\mathrm{e}}$ & $-4.6^{\mathrm{e}}$ & $-1.8^{\mathrm{a}}$ & $40.6^{\mathrm{a}}$ \\
$\mathrm{InP}$ & $1.34^{\mathrm{a}}$ & $0.21^{\mathrm{a}}$ & $3.12^{\mathrm{a}}$ & $0.15^{\mathrm{a}}$ & $5.0^{\mathrm{a}}$ & $5.87^{\mathrm{b}}$ & $2.24 \times 10^{-12 \mathrm{~d}}$ & $2.17 \times 10^{-12 \mathrm{~d}}$ & $-1.55^{\mathrm{e}}$ & $-4.4^{\mathrm{s}}$ & $-1.8^{\mathrm{g}}$ & $40.6^{\mathrm{g}}$ \\
\hline
\end{tabular}

a W. Richter, Springer Tracts in Modern Physics, Solid State Physics (Edited by G. Höhler), p. 121. Springer-Verlag (1976).

b G. Giesecke, Semiconductors and Semimetals Vol. 2 (Edited by R.K. Willardson \& A.C. Beer), p. 63. Academic Press, New York (1966).

c R. Weil \& W. Groves, J. Appl. Phys. 39, 4049 (1968).

d F. Hickernell \& W. Gayton, J. Appl. Phys. 37, 462 (1966).

e R. Humphreys, U. Rössler \& M. Cardona (to be published).

f A. Gavini \& M. Cardona, Phys. Rev. B1, 672 (1970).

B Values for Si from [8].

Table 2. Contributions to piezobirefringence of the various gaps of $\mathrm{GaP}$ and $\mathrm{InP}$ (units $10^{-11} \mathrm{~cm}^{2} /$ dyne)

\begin{tabular}{|c|c|c|c|c|c|c|c|}
\hline & \multicolumn{5}{|c|}{ Experimental } & \multicolumn{2}{|c|}{ Calculated } \\
\hline & $\underline{C_{0}}$ & $C_{0}^{\prime}$ & $C_{1}^{\prime}$ & $C_{2}$ & $C_{2}^{\prime}$ & $C_{1}$ & $\underline{C}_{1}^{\prime}$ \\
\hline $\mathrm{GaP}$ & -0.38 & -0.28 & 1.5 & 1.71 & -1.36 & -8 & 0.50 \\
\hline InP & -0.35 & -0.38 & - & 0.94 & 0.19 & -9 & 1.1 \\
\hline
\end{tabular}

photon energy for $\mathrm{GaP}$ and InP. The crosses represent values determined by conventional techniques and the dots values determined from the depolarization of the Raman spectrum. The full lines are fits to the experimental values according to the expressions given in the previous section. Because of the relatively weak dispersion of the $E_{1}$ and $E_{2}$ contributions in the region studied, it has been possible to determine only $C_{0}$ and $C_{0}^{\prime}$ from the experimental fits. Only in the case of $\mathrm{GaP} X \|[111]$ was it possible to obtain an unambiguous result for $C_{1}^{\prime}$. For the other fits we have used calculated values of $C_{1}$ and $C_{1}^{\prime}$ according to equations (10) and (12). The values of the constants entering into the calculations are summarized in Table 1 . In the evaluation of $C_{1}$ and $C_{1}^{\prime}$ we have used the values of $D_{3}^{3}, D_{1}^{5}$ and $D_{3}^{5}$ measured for Si [8] because these constants have not been measured for either GaP or InP.

Our experimental values of $C_{0}, C_{0}^{\prime}, C_{1}^{\prime}, C_{2}$ and $C_{2}^{\prime}$ are given in Table 2 together with the calculated values of $C_{1}$ and $C_{1}^{\prime}$. Our values of $C_{0}$ and $C_{0}^{\prime}$ allow $C_{0}^{\prime \prime}$ to be determined using equations (6) and (8). For $\mathrm{GaP}$ we obtain 6.2 and 5.5, for InP, 1.8 and 2.5. The difference between the values obtained for each substance are probably due to the simple model that has been used to fit the data.

However, it should be noted that the values obtained fall within the range of those determined both experimentally and theoretically for other materials of this family [2].

\section{REFERENCES}

1. J.F. Nye, Physical Properties of Crystals, p. 253. Clarendon Press, Oxford (1960).

2. For a review see M. Cardona, Atomic Structure and Properties of Solids (Edited by E. Burstein), p. 514. Academic Press, New York (1972).

3. C.W. Higginbotham, M. Cardona \& F.H. Pollak, Phys. Rev. 184, 821 (1969).

4. A. Shileika, M. Cardona \& F.H. Pollak, Solid State Commun. 7, 1113 (1969).

5. P.Y. Yu, F.H. Pollak \& M. Cardona, Phys. Rev. B3, 340 (1971).

6. P.Y. Yu \& M. Cardona, J. Phys. Chem. Solids 34, 29 (1973).

7. D.K. Biegelsen, J.C. Zesch \& C. Schwab, Phys. Rev. B14, 3578 (1976).

8. M. Chandrasekhar, M. Grimsditch \& M. Cardona, J.O.S.A. 68, 523 (1978); Phys. Rev. (in press).

9. See, for example, [3]

10. H. Vogelmann \& T. Fjeldly, Rev. Sci. Instrum. 45, 309 (1974).

11. W. Spitzer, M. Gershenzon, C. Frosch \& D. Gibbs, J. Phys. Chem. Solids 11, 339 (1959).

12. H.R. Philipp \& H. Ehenreich, Semiconductors and Semimetals, Vol. 3 (Edited by R.K. Willardson \& A.C. Beer), p. 93. Academic Press, New York (1967).

13. W. Bond, J. Appl. Phys. 36, 1674 (1965).

14. R. Dixon, J. Appl. Phys. 38, 5149 (1967).

15. B. Seraphin \& H. Bennett, Semiconductors and Semimetals Vol. 3 (Edited by R.K. Willardson \& A.C. Beer), p. 499. Academic Press, New York (1967).

16. The expressions for the birefringence due to the $E_{1}$ and $E_{1}+\Delta_{1}$ gaps given in [2] contains errors. These have been corrected as was already pointed out in [8]. 